\title{
THE VERTICAL STRUCTURE AND KINEMATICS OF THE GALAXY
}

\author{
C. SOUBIRAN \\ Centre d'Analyse de Images/MAMA \\ Observatoire de Paris \\ 61 Avenue de l'Observatoire \\ 75014 Paris, France
}

\begin{abstract}
A sample including 2370 stars with (U, V) velocities has been analyzed up to $z=2.5 \mathrm{kpc}$. It is shown that the observed vertical gradient in the velocity distribution can be explained by the sum of 3 discrete populations with constant kinematics. The observations are well fitted by exponential density laws for the thin disk and the thick disk with scale lengths of $280 \mathrm{pc}$ and $700 \mathrm{pc}$ respectively, and with local densities of $6 \%$ and $0.15 \%$ for the thick disk and halo respectively.
\end{abstract}

\section{Introduction}

The aim of this study is to analyze the mixture of the thin disk, thick disk and halo populations up to a few kpc above the plane, and from the deconvolution of their velocity distributions get some information about the kinematical parameters of the stellar populations, their vertical gradient, the relations between the thin disk and thick disk, the rotational velocity of the halo and the vertical density laws.

This project is conducted with Schmidt plates of different kinds digitized with the MAMA microdensitometer to provide proper motions and multicolour photometry for complete samples of stars on wide fields down to a limiting magnitude of about $\mathrm{V}=18$. Photometric distances are used to convert absolute proper motions into velocities.

A sample has already been obtained in a 7 square degree field in the direction of $M 3$ $\left(b=+80^{\circ}\right)$ from 11 plates on a time baseline of 40 years. Methods concerning the astrometric and photometric reductions, zero point of proper motions, distance estimation, etc. are described elsewhere (Soubiran 1992; Soubiran 1993a). The resulting accuracy of the proper motions is better than $2 \mathrm{mas} / \mathrm{yr}$, and the final sample includes 2370 stars with (U, V) velocities with respect to the Sun, and reaches $z=2.5 \mathrm{kpc}$.

\section{Kinematics of the Stellar Populations}

The kinematics of the sample versus $z$ distance above the plane has been presented in Soubiran (1993b). The U velocity is clustered around $0 \mathrm{~km} / \mathrm{s}$ at all distances whereas the asymmetric drift has a gradient of about $-40 \mathrm{~km} / \mathrm{s} / \mathrm{kpc}$. An increase of about $35 \mathrm{~km} / \mathrm{s} / \mathrm{kpc}$ in the dispersions is also observed, which is an expected feature and corresponds to the rising proportion of old stars with 
hotter kinematics as one looks far above the plane. The sample is a mixture of different populations with different kinematics and the question is to know if these populations have constant kinematics with $z$, in which case the vertical gradient is only due to the variation of their relative proportion, or if each population has its own vertical gradient. To answer this question one has to analyze separately the contribution of each population to the velocity distribution of the whole sample. This problem has been discussed previously by Nemec \& Nemec (1993).

The sample has been divided into 6 bins of distance, and in each bin of distance a fit has been performed with a non-informative method SEM to identify gaussian (U, V) components corresponding to the different populations. This algorithm is described in Celeux \& Diebolt (1986). The SEM solutions are presented in Fig. 1, on the $V$ velocity which is the most
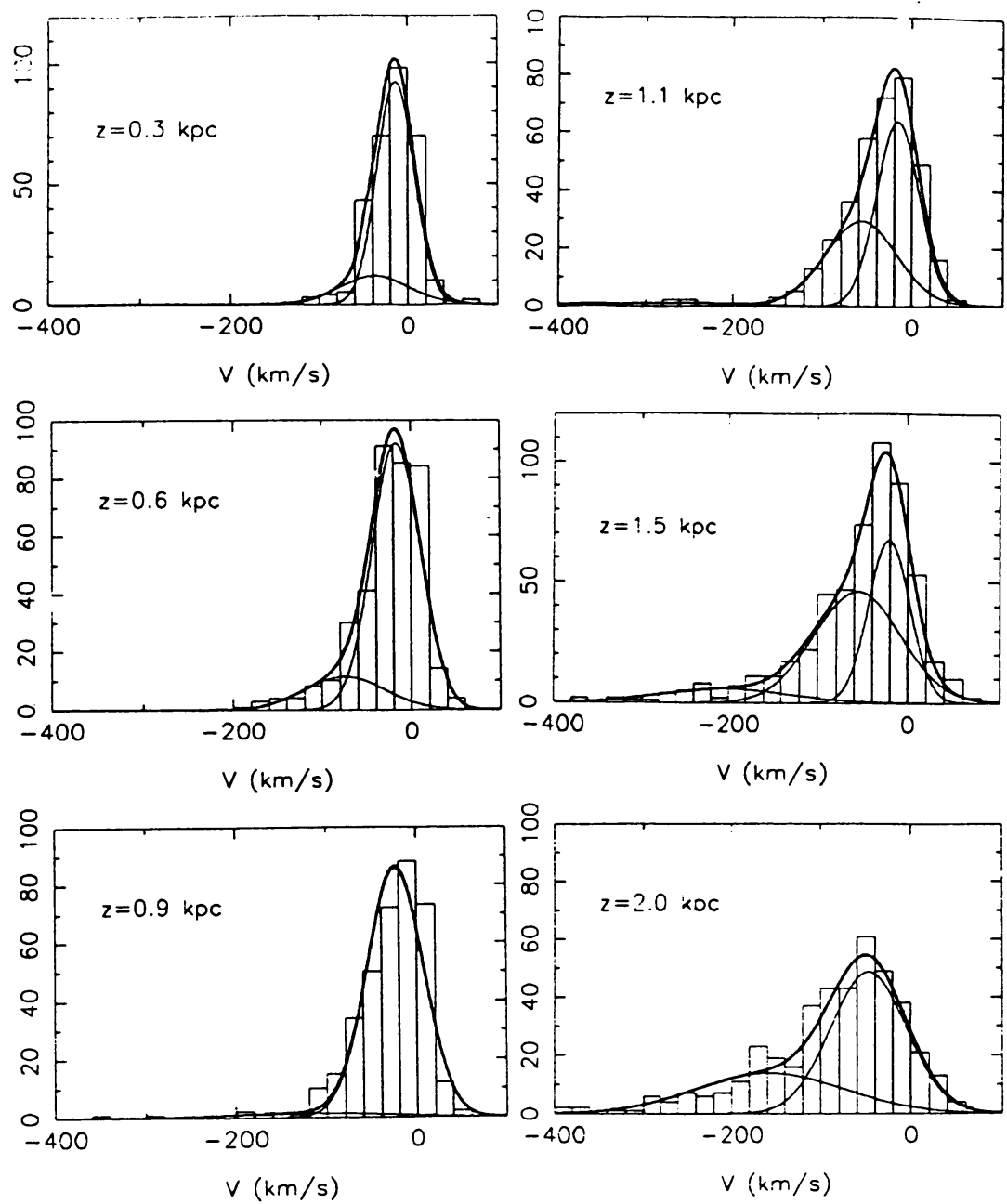

Figure 1. SEM deconvolution in gaussian components, presented on the $V$ histogram for 6 intervals of distance. 
discriminant variable.

A population with a low dispersion, corresponding to the thin disk, has been identified in 5 bins of distance up to $1.5 \mathrm{kpc}$, with no vertical gradient in its parameters. The kinematics of the thin disk appears to be the same at $0.5 \mathrm{kpc}$ and at $1.5 \mathrm{kpc}$. The weighted averages of the estimated parameters are:

$\bar{U}=3 \pm 4 \mathrm{~km} / \mathrm{s}, \bar{V}=-17 \pm 3 \mathrm{~km} / \mathrm{s}, \sigma_{U}=41 \pm 4 \mathrm{~km} / \mathrm{s}, \sigma_{V}=23 \pm 3 \mathrm{~km} / \mathrm{s}$.

The thick disk has also been identified in 5 bins of distance with no vertical gradient (Fig. 2). The estimated parameters have been averaged:

$\bar{U}=0 \pm 15 \mathrm{~km} / \mathrm{s}, \bar{V}=-52 \pm 16 \mathrm{~km} / \mathrm{s}, \sigma_{U}=56 \pm 11 \mathrm{~km} / \mathrm{s}, \sigma_{V}=43 \pm 6 \mathrm{~km} / \mathrm{s}$.

The kinematics of the halo has been deduced from the SEM deconvolution in the 3 highest bins of distance:

$\bar{U}=-4 \pm 8 \mathrm{~km} / \mathrm{s}, \bar{V}=-173 \pm 12 \mathrm{~km} / \mathrm{s}, \sigma_{U}=137 \pm 13 \mathrm{~km} / \mathrm{s}, \sigma_{V}=79 \pm 6 \mathrm{~km} / \mathrm{s}$. The mean asymmetric drift leads to a rotational velocity of $V_{\text {rot }}=58 \pm 12 \mathrm{~km} / \mathrm{s}$.
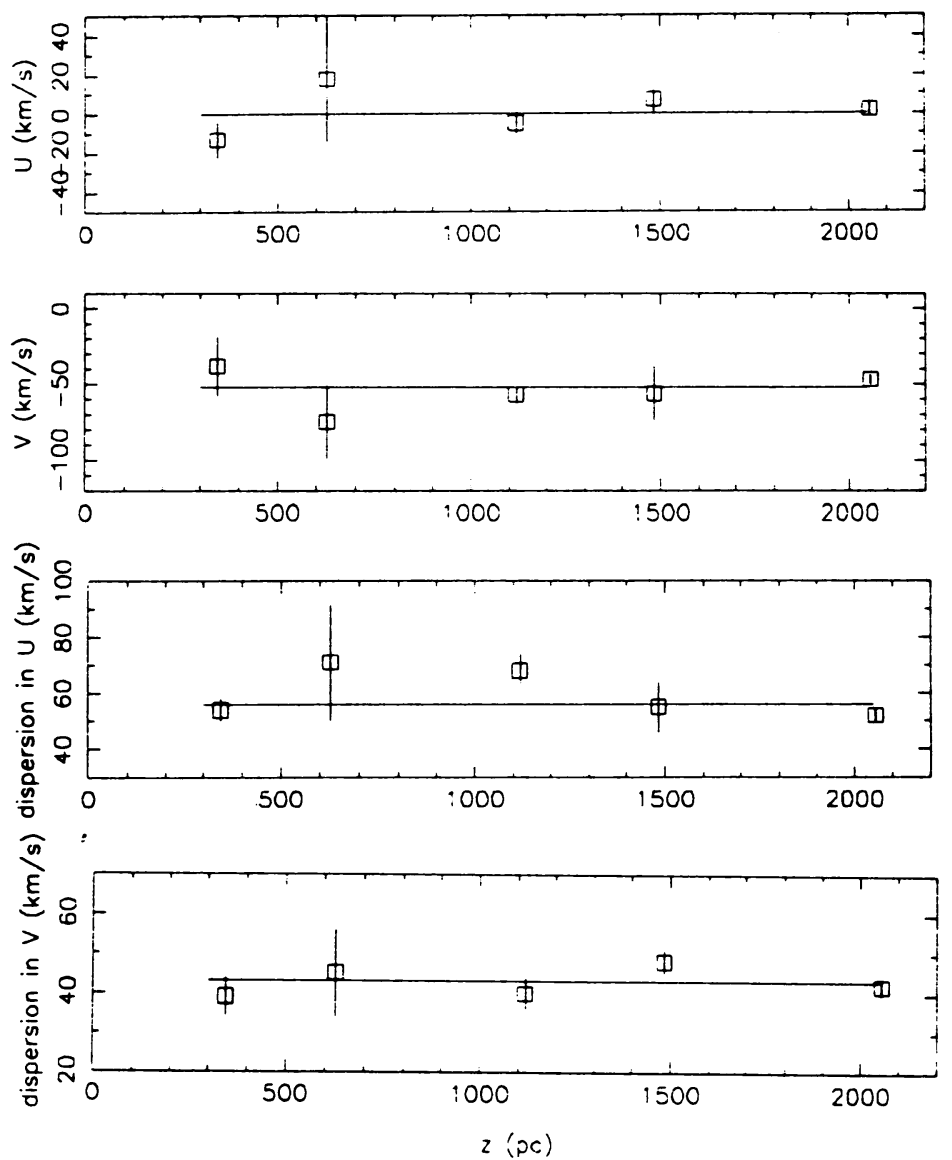

Figure 2. Kinematics of the thick disk versus $z$ distance. The horizontal planes indicate the weighted average for each parameter. 


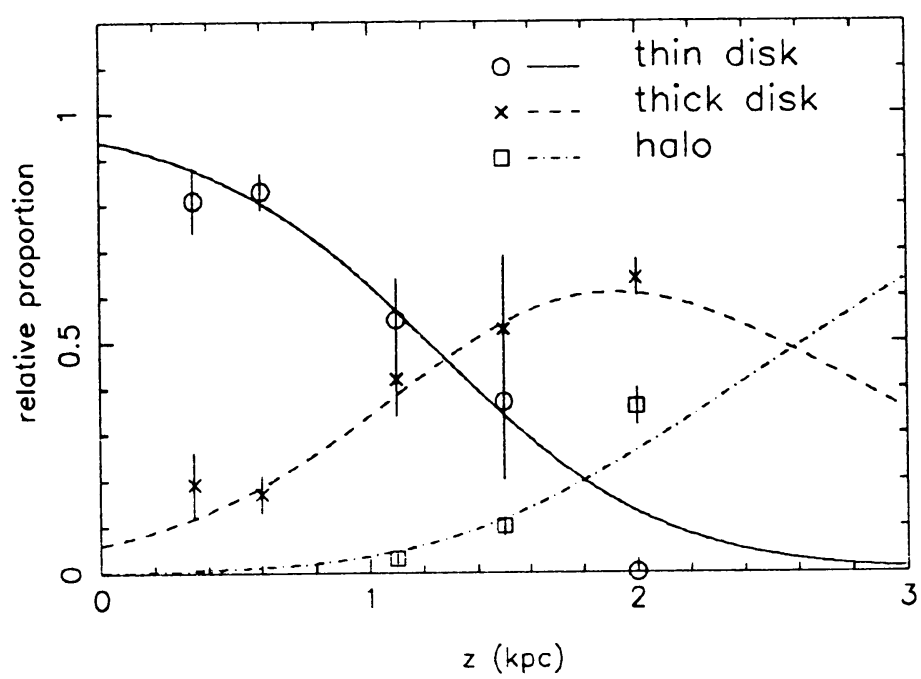

Figure 3. Ratio of the 3 stellar populations versus $z$ distance. The points indicate the SEM solutions (circles for the thin disk, cross for the thick disk, squares for the halo). The curves are the result of exponential density laws with scale lengths of $280 \mathrm{pc}$ and $700 \mathrm{pc}$ for the thin disk and thick disk respectively, and with local densities of $6 \%$ and $0.15 \%$ for the thick disk and halo respectively.

\section{Vertical Density Laws}

The SEM deconvolution provides in each bin of distance an estimate of the relative fraction of the different populations. These estimates are plotted in Fig. 3, representing the ratio of the 3 stellar populations versus $z$. The points are well fitted with exponential density laws for the thin and thick disks, with scale heights of $280 \mathrm{pc}$ and $700 \mathrm{pc}$ respectively, and local densities of $6 \%$ and $0.15 \%$ for the thick disk and halo. The density law of the halo is supposed to be independent of $z$ up to $2 \mathrm{kpc}$. At $2 \mathrm{kpc}$, this model predicts a ratio of $10 \%$ for the thin disk stars, whereas no such stars have been found in the data at this distance. But this may be due to the very small proportion of the expected gaussian component, which completely overlaps the thick disk population (see in Fig. 1, the histogram of $\mathrm{V}$ at $z=2 \mathrm{kpc}$ ). In this situation of overlapping components and extreme proportions, SEM has not been able to deconvolve the thin disk.

The scale height of the thick disk is a subject of controversy. There is a large range of determinations. The low value of $h_{z}=700 \mathrm{pc}$ is in agreement with Robin (1993) and Von Hippel \& Bothun (1993), but recent papers also quote values around $1.5 \mathrm{kpc}$ (see for example Morrison 1993, or Majewski 1993).

To verify the consistency of the results obtained at the different steps, the $(U, V)$ distribution of a mixture of 3 discrete populations with constant kinematics has been simulated and compared to the observed distribution (Fig. 4). Observations and simulation fit very well. 

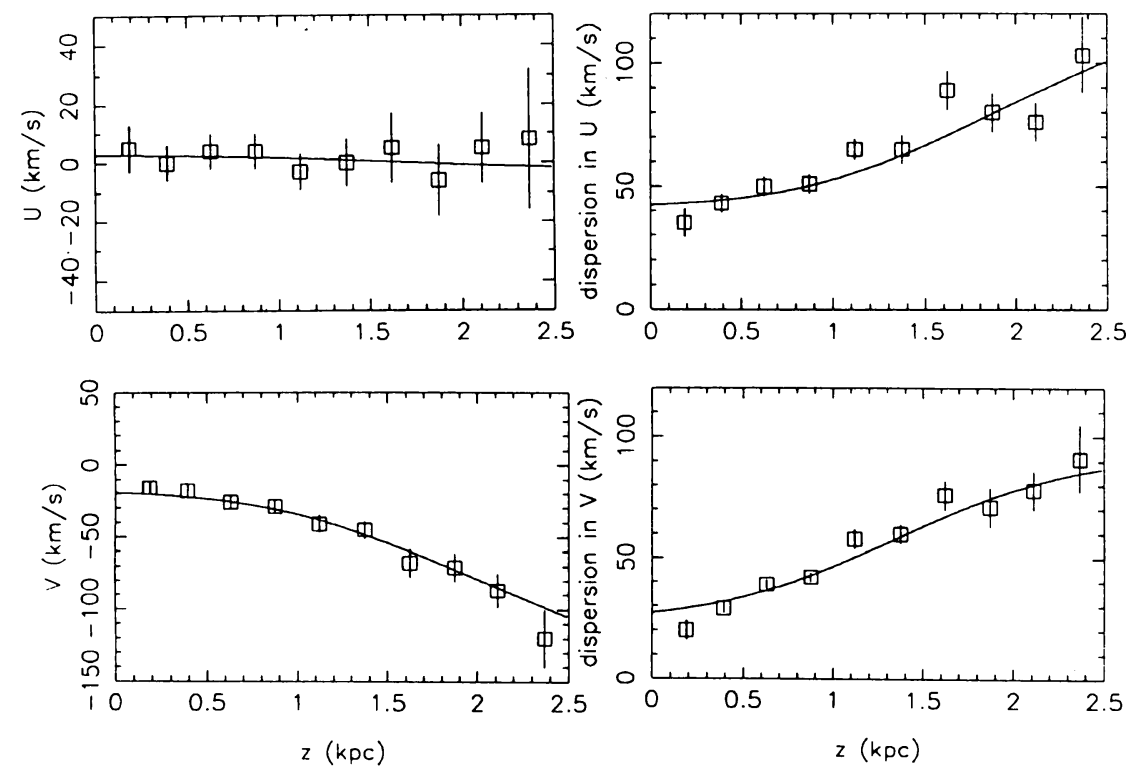

Figure 4. Comparison between the observed (U, V) distribution of the whole sample (squares) and the sum of 3 discrete populations, simulated with constant kinematics as indicated in section 2 and with density laws as indicated in section 3 .

\section{Future Work}

A program of spectroscopic observations for a part of this sample has now begun. Already 100 spectra have been measured, and 500 more spectra are expected by next year. These new data will complete this study in including the $\mathrm{W}$ velocity and the metallicity distribution.

\section{References}

Celeux, G. and Diebolt, J., 1986. Ref. Statistique Appliquée, 34, 35.

Morrison, H.L., 1993. Astron. J., 105, 539.

Majewski, S.R., 1993. This volume.

Nemec, J.M. and Nemec, A.F.L., 1993. Astron. J., 105, 1455.

Robin, A., 1993. These proceedings.

Soubiran, C., 1992. Astron. Astrophys., 259, 394.

Soubiran, C., 1993a. Astron. Astrophys., 274, 181.

Soubiran, C., 1993b. In 'Galactic and Solar System Optical Astrometry', eds. L. Morrison and

G. Gilmore, Cambridge University Press (in press).

von Hippel, T. and Bothun, G.D., 1993. Astrophys. J., 407, 115. 